\title{
The Non-symplectic Index of Supersingular K3 Surfaces
}

\author{
Junmyeong Jang
}

\begin{abstract}
In this paper, we find the non-symplectic indexes of all supersingular K3 surfaces in terms of their period spaces when the base field is of odd characteristic. And we show that the maximal non-symplectic index of a K3 surface defined over a field of odd characteristic $p$ is $p^{10}+1$.
\end{abstract}

\section{Introduction}

Let $k$ be an algebraically closed field. Given a K3 surface over $k$, the non-symplectic index $N_{X}$ of $X$ is defined to be the order of the image of the natural homomorphism

$$
\rho_{X}: \operatorname{Aut}(X) \rightarrow \operatorname{GL}\left(H^{0}\left(X, \Omega_{X / k}^{2}\right)\right)
$$

In this paper, we deal with supersingular K3 surfaces (for definition, see Section 2 below) and determine their non-symplectic indices:

Theorem 1.1. Let $k$ be an algebraically closed field of odd characteristic $p$ and $X$ be a supersingular $K 3$ surface of Artin invariant $\sigma$ over $k$. Then the non-symplectic index $N_{X}$ of $X$ is given by $N_{X}=p^{m}+1$ where $m=0$ or a positive integer such that $\sigma / m$ is an odd integer. Given $\sigma$ and $m$ as above, the supersingular K3 surfaces of Artin invariant $\sigma$ and non-symplectic index $p^{m}+1$ form a family of dimension $\sigma-1$ (if $m=0$ ) or $(\sigma-m) /(2 m)$ (if $m>0$ ). When $m$ attains the maximum $\sigma$ so that the non-symplectic index is $p^{\sigma}+1$, $X$ is unique up to isomorphisms.

Recalling that $\sigma$ is a positive integer up to 10 , we can summarize our result in the following table.

Received September 13, 2018; Accepted December 3, 2018.

Communicated by Yoichi Miyaoka.

2010 Mathematics Subject Classification. 14J20, 14J28.

Key words and phrases. supersingular K3 surface, non-symplectic index, crystalline Torelli theorem. 


\begin{tabular}{|c|c|c|}
\hline$\sigma$ & $\begin{array}{c}\text { non-symplectic } \\
\text { index of } X\end{array}$ & family \\
\hline 1 & $p+1$ & unique \\
\hline 2 & 2 & generic \\
& $p^{2}+1$ & unique \\
\hline 3 & 2 & generic \\
& $p+1$ & 1 dimensional \\
4 & $p^{3}+1$ & unique \\
\hline \multirow{3}{*}{5} & 2 & generic \\
& $p^{4}+1$ & unique \\
\hline & 2 & generic \\
& $p^{5}+1$ & 2 dimensional \\
& 2 & unique \\
\hline & $p^{2}+1$ & generic \\
$p^{6}+1$ & dimensional \\
& & unique \\
\hline
\end{tabular}

\begin{tabular}{|c|c|c|}
\hline$\sigma$ & $\begin{array}{c}\text { non-symplectic } \\
\text { index of } X\end{array}$ & family \\
\hline 7 & 2 & generic \\
& $p+1$ & 3 dimensional \\
& $p^{7}+1$ & unique \\
\hline 8 & 2 & generic \\
& $p^{8}+1$ & unique \\
\hline 9 & 2 & generic \\
& $p^{3}+1$ & 1 dimensional \\
& $p^{9}+1$ & dimensional \\
& 2 & unique \\
\hline 10 & $p^{2}+1$ & 2 dimensional \\
& $p^{10}+1$ & unique \\
\hline
\end{tabular}

Table 1.1

The present paper is organized as follows. In Section 2, we quickly survey the background of the problem and basic notions. In Section 3, we review the classification of supersingular K3 surfaces and the crystalline Torelli theorem, which are the main tools in the proof of the main theorem. The proof of the main theorem is completed in Section 4. In Section 5, we give explicit models over $\mathbb{F}_{p}$ of some special supersingular K3 surfaces, that is, supersingular K3 surfaces with Artin invariant $\sigma$ and non-symplectic index $p^{\sigma}+1$.

\section{Background of the problem}

For an algebraic complex K3 surface $X$, the second integral singular cohomology of $X$, $H^{2}(X, \mathbb{Z})$ is a free $\mathbb{Z}$-module of rank 22 equipped with an even unimodular lattice structure of signature $(3,19)$. This lattice is unique up to isomorphism and is isomorphic to $U^{\oplus 3} \oplus E_{8}^{\oplus 2}$. Here $U$ is the unimodular hyperbolic lattice of rank 2 and $E_{8}$ is the unimodular root lattice of signature $(0,8)$. The cycle map gives a primitive lattice embedding $N S(X) \hookrightarrow H^{2}(X, \mathbb{Z})$. The Picard number of $X$ is the rank of $N S(X)$. We say the or- 
thogonal complement of this embedding is the transcendental lattice of $X$ and we denote the transcendental lattice by $T(X)$. Then we have

$$
\operatorname{rank} T(X)=22-\operatorname{rank} N S(X) \leq 21
$$

It is well known that $\operatorname{Im} \rho_{X}$ is a finite cyclic group and that the rank of $T(X)$ is divisible by $\phi\left(N_{X}\right)$. Here $\phi$ is the Euler totient function. It follows that

$$
\phi\left(N_{X}\right) \leq \operatorname{rank} T(X) \leq 21 \text { and } \quad N_{X} \leq 66
$$

Example 2.1. Consider a complex elliptic K3 surface $X_{66}$ given as the minimal model of

$$
y^{2}=x^{3}+t\left(t^{11}-1\right)
$$

and an automorphism of $X_{66},($ see $[12)$

$$
\alpha_{66}:(x, y, t) \mapsto\left(\xi_{66}^{40} x, \xi_{66}^{27} y, \xi_{66}^{54} t\right), \quad \xi_{66}=e^{2 \pi i / 66} .
$$

The order of $\rho_{X_{66}}\left(\alpha_{66}\right)$ is 66 and $N_{X_{66}}=66$. Therefore the maximal non-symplectic index of a complex algebraic K3 surface is 66 .

Remark 2.2. When $N$ is a positive integer satisfying $\phi(N) \leq 21$ and $N \neq 60$, there exists a complex K3 surface $X$ and an automorphism $\alpha \in \operatorname{Aut}(X)$, such that the ord $\rho_{X}(\alpha)=N$ (see [2, pp. 18-19], [11, Example 3.1]).

Assume that $k$ is an algebraically closed field of odd characteristic $p, W$ is the ring of Witt vectors of $k$ and $K$ is the fraction field of $W$. Assume $X$ is a K3 surface defined over $k$. The height of the formal Brauer group of $X, \widehat{\operatorname{Br}}_{X}$ is an integer between 1 and 10 or infinite. The height of $X$ is the height of the formal Brauer group $\widehat{\operatorname{Br}}_{X}$. If $X$ is of finite height $h$, the Picard number of $X$ is at most $22-2 h$ (see [6, Proposition II.5.12]). If the height of $X$ is infinite, we call $X$ is a supersingular K3 surface. The Picard number of a supersingular K3 surface is 22.

The second crystalline cohomology of $X, H_{\text {cris }}^{2}(X / W)$ is a free $W$-module of rank 22 equipped with a canonical Frobenius semi-linear endomorphism $\mathbf{F}: H_{\text {cris }}^{2}(X / W) \rightarrow$ $H_{\text {cris }}^{2}(X / W)$ and a unimodular $W$-lattice structure. The cycle map gives an embedding of $W$-lattices $N S(X) \otimes W \hookrightarrow H_{\text {cris }}^{2}(X / W)$. If the height of $X$ is finite, this embedding is primitive and the orthogonal complement of $N S(X) \otimes W$ in $H_{\text {cris }}^{2}(X / W)$ is called the crystalline transcendental lattice which is denoted by $T_{\text {cris }}(X)$. In a previous work (see [10]), we prove that, as the case of complex K3 surfaces, for a K3 surface $X$ of finite height over $k$, the $\phi$-value of the non-symplectic index, $\phi\left(N_{X}\right)$ divides the rank of $T_{\text {cris }}(X)$. Therefore $\phi\left(N_{X}\right) \leq 21$ and the non-symplectic index of a K3 surface of finite height over $k$ is at most 66 . 
Remark 2.3. Assume $p$ does not divide 66. There is a $\mathrm{K} 3$ surface $X$ of finite height with $N_{X}=66$ if and only if $p^{m} \not \equiv-1(\bmod 66)$ for any positive integer $m$ (see 9 , Corollary 4.3]).

Assume $X$ is a supersingular K3 surface over $k$. Then $N S(X)$ is an even lattice of signature $(1,21)$. The discriminant group $l(N S(X))=\operatorname{Hom}(N S(X), \mathbb{Z}) / N S(X)$ is isomorphic to $(\mathbb{Z} / p)^{2 \sigma}$ for an integer $\sigma$ between 1 and 10 . We say $\sigma$ is the Artin invariant of $X$. The Neron-Severi lattice of a supersingular K3 surface is uniquely determined by the base characteristic $p$ and the Artin invariant $\sigma$ (see [13, Proposition 1.14.1]). We denote this unique lattice by $N_{p, \sigma}$. Note that the class number of $N_{p, \sigma}$ is 1 (loc. cit.). All the supersingular K3 surfaces of Artin invariant $\sigma$ form a $\sigma-1$ dimensional family and a supersingular K3 surface of Artin invariant 1 is unique up to isomorphism. For the non-symplectic index of a supersingular K3 surface, the following is known.

Theorem 2.4. [14] When $X$ is a supersingular K3 surface of Artin invariant $\sigma$ over $k$, the non-symplectic index $N_{X}$ divides $p^{\sigma}+1$.

In particular, $N_{X}$ is finite. In a previous work, we prove that the non-symplectic index of a K3 surface of Artin invariant 1 over $k$ is exactly $p+1$ (see [7]). The proof of that result is based on the crystalline Torelli theorem.

Remark 2.5. In [7], as a corollary of the main theorem, we show that, if $\phi(p+1)>20$, then there exists an automorphism of a supersingular K3 surface of Artin invariant 1 which cannot be lifted over a ring of characteristic 0. After work of several people, now it is known that every supersingular K3 surface contains an automorphism which cannot be lifted over a ring of characteristic 0 (see $3,5,18,20]$ ).

In the proof of Theorem 1.1, we will see the non-symplectic index of all supersingular K3 surfaces is determined by the period space (see Section 3 ). This generalizes the previous result for a K3 surface of Artin invariant 1.

\section{Strictly characteristic subspaces and crystalline Torelli theorem}

In this section, we review Ogus' classification of supersingular K3 surfaces and the crystalline Torelli theorem in 15, 16.

Assume $k$ is an algebraically closed field of odd characteristic $p$. We fix an integer $\sigma$ between 1 and 10. The discriminant group $l\left(N_{p, \sigma}\right)$ is a $2 \sigma$-dimensional vector space over the prime field $\mathbb{F}_{p}$ equipped with the induced quadratic form. The discriminant of this quadratic form is $(-1)^{\sigma} \Delta$ where $\Delta$ is a quadratic non-residue modulo $p$. Hence $l\left(N_{p, \sigma}\right)$ does not have a $\sigma$-dimensional isotropic subspace over $\mathbb{F}_{p}$. We set

$$
f=\mathrm{id} \otimes F_{k}: l\left(N_{p, \sigma}\right) \otimes k \rightarrow l\left(N_{p, \sigma}\right) \otimes k .
$$


Here $F_{k}$ is the Frobenius morphism of $k$. A $\sigma$-dimensional isotropic subspace of $l\left(N_{p, \sigma}\right) \otimes k$, denoted by $\mathcal{K}$ is a characteristic subspace if $\mathcal{K}+f(\mathcal{K})$ is $\sigma+1$ dimensional. A characteristic subspace $\mathcal{K}$ is a strictly characteristic subspace if $\mathcal{K} \cap l\left(N_{p, \sigma}\right)=0$ in $l\left(N_{p, \sigma}\right) \otimes k$. This is equivalent to the condition that $\{x \in \mathcal{K} \mid f(x)=x\}=0$. Assume $\mathcal{K}$ is a strictly characteristic subspace of $l\left(N_{p, \sigma}\right) \otimes k$. Then it is easy to see that $l_{\mathcal{K}}=\bigcap_{i=0}^{\sigma-1} f^{-i} \mathcal{K}$ is a line in $l\left(N_{p, \sigma}\right) \otimes k$. Moreover we have

$$
\sum_{i=0}^{\sigma-1} f^{i}\left(l_{\mathcal{K}}\right)=\mathcal{K} \quad \text { and } \quad \sum_{i=0}^{2 \sigma-1} f^{i}\left(l_{\mathcal{K}}\right)=l\left(N_{p, \sigma}\right) \otimes k .
$$

Let $v$ be a non zero vector in $l_{\mathcal{K}}$ and we denote $v_{i}=f^{i-1}(v)$. Then $\left\{v_{1}, \ldots, v_{\sigma}\right\}$ is a basis of $\mathcal{K}$ and $\left\{v_{1}, \ldots, v_{2 \sigma}\right\}$ is a basis of $l\left(N_{p, \sigma}\right) \otimes k$. Since the pairing of $l\left(N_{p, \sigma}\right) \otimes k$ is defined over $\mathbb{F}_{p}, f(\mathcal{K})$ is an isotropic subspace of $l\left(N_{p, \sigma}\right) \otimes k$. If $v_{1} \cdot v_{\sigma+1}=0$, then the subspace generated by $v_{1}, \ldots, v_{\sigma+1}$ is an isotropic subspace of dimension $\sigma+1$. Because $l\left(N_{p, \sigma}\right)$ is non-degenerate, it is impossible and $v_{1} \cdot v_{\sigma+1} \neq 0$. After a suitable scalar multiplication, we may assume $v_{1} \cdot v_{\sigma+1}=1$. Note that $v_{1}$ is uniquely determined up to multiplication by $\left(p^{\sigma}+1\right)$-th roots of unity. Now we put $a_{i}=v_{1} \cdot v_{\sigma+1+i} \in k(i=1, \ldots, \sigma-1)$. Because the pairing on $l\left(N_{p, \sigma}\right) \otimes k$ is defined over $\mathbb{F}_{p}$, the intersection matrix of $l\left(N_{p, \sigma}\right)$ in terms of the basis $v_{1}, \ldots, v_{2 \sigma}$ is $\left(\begin{array}{cc}0 & A \\ A^{t} & 0\end{array}\right)$, where

$$
A=\left(\begin{array}{cccccc}
1 & a_{1} & a_{2} & a_{3} & \cdots & a_{\sigma-1} \\
0 & 1 & F_{k}\left(a_{1}\right) & F_{k}\left(a_{2}\right) & \cdots & F_{k}\left(a_{\sigma-2}\right) \\
0 & 0 & 1 & F_{k}^{2}\left(a_{1}\right) & \cdots & F_{k}^{2}\left(a_{\sigma-3}\right) \\
\vdots & \vdots & \vdots & \vdots & & \vdots \\
0 & 0 & 0 & 0 & \cdots & 1
\end{array}\right) .
$$

If we replace $v_{1}$ by $\xi v_{1}$, for a $p^{\sigma}+1$-th root of unity $\xi$, then $a_{i}$ is replaced by $\xi^{p^{\sigma+i}+1} a_{i}$. Let $\mathcal{M}$ be the set of $O\left(l\left(N_{p, \sigma}\right)\right)$-conjugacy classes of strictly characteristic subspaces of $l\left(N_{p, \sigma}\right) \otimes k$. By the above observation, we have a map

$$
\Psi: \mathcal{M} \rightarrow \mathbb{A}^{\sigma-1} / \mu_{p^{\sigma}+1}(k), \quad \mathcal{K} \mapsto\left(a_{1}, \ldots, a_{\sigma-1}\right)
$$

Theorem 3.1. 15. Theorem 3.21] The map $\Psi$ is a bijection from $\mathcal{M}$ to $\mathbb{A}^{\sigma-1} / \mu_{p^{\sigma}+1}(k)$.

Assume $X$ is a supersingular K3 surface of Artin invariant $\sigma$ over $k$. We fix an isometry $N S(X) \simeq N_{p, \sigma}$. The cycle map $N S(X) \otimes W \hookrightarrow H_{\text {cris }}^{2}(X / W)$ is an embedding of $W$-lattices of the same rank, so we have the following chain

$$
N_{p, \sigma} \otimes W \subset H_{\text {cris }}^{2}(X / W) \subset N_{p, \sigma}^{*} \otimes W \subset N_{p, \sigma} \otimes K
$$


The cokernel $H_{\text {cris }}^{2}(X / W) /\left(N_{p, \sigma} \otimes W\right)$ is a $\sigma$-dimensional isotropic $k$-subspace of

$$
\left(N_{p, \sigma}^{*} \otimes W\right) /\left(N_{p, \sigma} \otimes W\right)=l\left(N_{p, \sigma}\right) \otimes k .
$$

We denote $H_{\text {cris }}^{2}(X / W) /\left(N_{p, \sigma} \otimes W\right)$ by $\mathcal{K}(X)$. We say $\mathcal{K}(X)$ is the period space of $X$.

Theorem 3.2. [15, Theorem 3.20] For a supersingular K3 surface $X, \mathcal{K}(X)$ is a strictly characteristic subspace of $l\left(N_{p, \sigma}\right) \otimes k$.

Note that the position of $\mathcal{K}(X)$ in $l\left(N_{p, \sigma}\right) \otimes k$ depends on the choice of $N S(X) \simeq N_{p, \sigma}$. But the class of $\mathcal{K}(X)$ in $\mathcal{M}$ does not depend on the choice of the isometry.

Remark 3.3. In [15], $\mathcal{K}(X)$ is defined as $f^{-1}\left(H_{\text {cris }}^{2}(X / W) / N S(X) \otimes W\right)$. For simplicity, we use a slightly different definition. All the arguments in this paper are valid for both definitions.

We put $\Delta=\left\{v \in N_{p, \sigma} \mid v \cdot v=-2\right\}$. For any $v \in \Delta$, let

$$
s_{v}: w \mapsto w+(v \cdot w) v \in O\left(N_{p, \sigma}\right)
$$

be the reflection along the line of $v$. The Weyl group of $N_{p, \sigma}$, which is denoted by $W_{N_{p, \sigma}}$, is the subgroup of $O\left(N_{p, \sigma}\right)$ generated by $s_{v}(v \in \Delta)$ and - id. The real quadratic space $N_{p, \sigma} \otimes \mathbb{R}$ is of signature $(1,21)$. Let $\mathcal{P}=\left\{v \in N_{p, \sigma} \otimes \mathbb{R} \mid v \cdot v>0\right\}$ be the positive cone of $N_{p, \sigma} \otimes \mathbb{R}$. The positive cone $\mathcal{P}$ has two connected components. Let $\mathcal{C}$ be the set of connected components of $\mathcal{P}-\bigcup_{v \in \Delta}\langle v\rangle^{\perp}\left(\subset N_{p, \sigma} \otimes \mathbb{R}\right)$. The ample cone of $X$, which is denoted by $\mathcal{A}_{X}$, is an element of $\mathcal{C}$. The action of the Weyl group $W_{N_{p, \sigma}}$ on $\mathcal{C}$ is simple and transitive (see $[16,1.10])$. We denote the representations of $\operatorname{Aut}(X)$ on $N_{p, \sigma}$ and $l\left(N_{p, \sigma}\right)$ by

$$
\lambda_{X}: \operatorname{Aut}(X) \rightarrow O\left(N_{p, \sigma}\right) \text { and } \nu_{X}: \operatorname{Aut}(X) \rightarrow O\left(l\left(N_{p, \sigma}\right)\right) .
$$

For an automophism $\alpha \in \operatorname{Aut}(X), \lambda_{X}(\alpha)$ preserves $\mathcal{A}_{X}$ and $\nu_{X}(\alpha)$ preserves $\mathcal{K}(X)$.

Theorem 3.4 (crystalline Torelli theorem). 16, Theorems II, III], 1, Theorem 5.1.9]

(1) Let $g \in O\left(N_{p, \sigma}\right)$. If $g \otimes \mathbb{R}$ preserves $\mathcal{A}_{X}$ and $\left.g\right|_{l\left(N_{p, \sigma}\right)}$ preserves $\mathcal{K}(X)$, then there exists a unique $\alpha \in \operatorname{Aut}(X)$ such that $\lambda_{X}(\alpha)=g$.

(2) If $\mathcal{K}$ is a strictly characteristic space of $l\left(N_{p, \sigma}\right)$, there exists a unique supersingular $K 3$ surface $X$ of Artin invariant $\sigma$ over $k$ up to isomorphism such that the class of $\mathcal{K}(X)$ is equal to the class of $\mathcal{K}$ in $\mathcal{M}$.

By Theorem 3.1 and Theorem 3.4(2), the isomorphism classes of supersingular K3 surfaces of Artin invariants $\sigma$ over $k$ are classified by $\mathbb{A}^{\sigma-1} / \mu_{p^{\sigma}+1}(k)$.

The two representations of $\operatorname{Aut}(X), \rho_{X}$ and $\nu_{X}$, have the same kernel, so $\operatorname{Im} \rho_{X} \simeq$ $\operatorname{Im} \nu_{X}$ (see [14, Theorem 2.1]). In particular, $N_{X}=\left|\operatorname{Im} \nu_{X}\right|$. Indeed, for any $\alpha \in \operatorname{Aut}(X)$, 
$\nu_{X}(\alpha)$ is rational over $\mathbb{F}_{p}$ and preserves $\mathcal{K}(X)$, so $\nu_{X}(\alpha)\left(l_{\mathcal{K}(X)}\right)=l_{\mathcal{K}(X)}$. When $v_{1}, \ldots, v_{2 \sigma}$ are a basis of $l\left(N_{p, \sigma}\right) \otimes k$ defined above associated to the strictly characteristic subspace $\mathcal{K}(X)$, then every $v_{i}$ is an eigenvector of $\nu_{X}(\alpha)$. If $\nu_{X}(\alpha)\left(v_{1}\right)=\zeta v_{1}, \nu_{X}(\alpha)\left(v_{i}\right)=\zeta p^{i-1} v_{i}$ for all $i=1, \ldots, 2 \sigma$. Therefore, $\nu_{X}(\alpha)=\mathrm{id}$ if and only if $\nu_{X}(\alpha)\left(v_{1}\right)=v_{1}$ if and only if $\nu_{X}(\alpha)\left(v_{i}\right)=v_{i}$ for any $i=1, \ldots, 2 \sigma$. The character of $\operatorname{Aut}(X)$ on $H^{0}\left(X, \Omega_{X / k}^{2}\right)$, which is just the representation $\rho_{X}$, is isomorphic to the character of $\operatorname{Aut}(X)$ on the 1-dimensional $k$-space generated by $v_{2 \sigma}$ (see $[15,3.20 .1]$ ) and the claim follows. In particular, $\operatorname{Im} \nu_{X}$ is a finite cyclic group.

Lemma 3.5. Assume $g \in O\left(l\left(N_{p, \sigma}\right)\right)$ preserves $\mathcal{K}(X)$. Then there exists $\alpha \in \operatorname{Aut}(X)$ such that $\nu_{X}(\alpha)=g$.

Proof. Because the signature of $N_{p, \sigma}$ is $(1,21)$ and the dimension as an $(\mathbb{Z} / p)$-space of $l\left(N_{p, \sigma}\right)$ is $2 \sigma \leq 20=22-2$, the class number of $N_{p, \sigma}$ is 1 and the reduction map $O\left(N_{p, \sigma}\right) \rightarrow O\left(l\left(N_{p, \sigma}\right)\right)$ is surjective (see 13, Theorem 1.14.2]). There exists $\mathfrak{g} \in O\left(N_{p, \sigma}\right)$ such that $\left.\mathfrak{g}\right|_{l\left(N_{p, \sigma}\right)}=g$. Let $\mathfrak{g}\left(\mathcal{A}_{X}\right)=\mathcal{B} \in \mathcal{C}$.

If $\mathcal{B}$ is in the same connected component of $\mathcal{P}_{X}$ with $\mathcal{A}_{X}$, the unique $\varphi \in W_{N_{p, \sigma}}$, satisfying $\varphi(\mathcal{B})=\mathcal{A}_{X}$, can be written as $\varphi=s_{w_{1}} \circ s_{w_{2}} \circ \cdots \circ s_{w_{n}}$ for $w_{i} \in \Delta$. Let $M_{i}$ be the rank 1 sub lattice of $N_{p, \sigma}$ generated by $w_{i}$. Then $l\left(M_{i}\right)=\mathbb{Z} / 2$ and $l\left(M_{i}^{\perp}\right)=\mathbb{Z} / 2 \oplus l\left(N_{p, \sigma}\right)$. Since $\left.s_{w_{i}}\right|_{M_{i}^{\perp}}=\mathrm{id}$, it follows $\left.s_{w_{i}}\right|_{l\left(N_{p, \sigma}\right)}=\mathrm{id}$. It follows that $\varphi \circ \mathfrak{g}$ preserves $\mathcal{A}_{X}$ and $\mathcal{K}(X)$, so, by the crystalline Torelli theorem, $\varphi \circ \mathfrak{g} \in \operatorname{Aut}(X)$ and $\nu(\varphi \circ \mathfrak{g})=g$.

Now assume $\mathcal{B}$ is in the other connected component of $\mathcal{P}$ with $\mathcal{A}_{X}$. Since the signature of $N_{p, \sigma}$ is $(1,21), N_{p, \sigma} \otimes \mathbb{R}$ contains a vector of self intersection 2. If $q$ is a prime number different from $p, N_{p, \sigma} \otimes \mathbb{Z}_{q}$ is even unimodular of rank 22, so it contains a vector of self intersection 2. The length of the unimodular part of $N_{p, \sigma} \otimes \mathbb{Z}_{p}$ is $22-2 \sigma \geq 2$, so $N_{p, \sigma} \otimes \mathbb{Z}_{p}$ has a vector of self intersection 2. By the Hasse principle, there exists a lattice in the genus of $N_{p, \sigma}$ which contains a vector of self intersection 2. But the class number of $N_{p, \sigma}$ is 1 , so $N_{p, \sigma}$ itself contains a vector $u$ such that $u \cdot u=2$. Let

$$
t_{u}: w \mapsto w-(w \cdot u) u \in O\left(N_{p, \sigma}\right)
$$

be the reflection along the line of $u$. Then $t_{u}$ exchanges the two connected components of $\mathcal{P}$ and $\left.t_{u}\right|_{l\left(N_{p, \sigma}\right)}=\mathrm{id}$. And $\mathcal{B}^{\prime}=t_{u}(\mathcal{B})$ is in the same connected component of $\mathcal{P}_{X}$ with $\mathcal{A}_{X}$. The unique $\varphi \in W_{N_{p, \sigma}}$, satisfying $\varphi\left(\mathcal{B}^{\prime}\right)=\mathcal{A}_{X}$, can be expressed as a composition of reflections along -2 vectors and $\left.\varphi\right|_{l\left(N_{p, \sigma}\right)}=\mathrm{id}$ as in the above case. Again, by the crystalline Torelli theorem, $\varphi \circ t_{u} \circ \mathfrak{g} \in \operatorname{Aut}(X)$ and $\nu\left(\varphi \circ t_{u} \circ \mathfrak{g}\right)=g$.

Corollary 3.6. The non-symplectic index of $X$ is even.

Proof. Since - id $\in O\left(l\left(N_{p, \sigma}\right)\right)$ preserves every subspace of $l\left(N_{p, \sigma}\right) \otimes k,-\mathrm{id} \in \operatorname{Im} \nu_{X}$ and the order of $-\mathrm{id}$ is 2 . 


\section{Proof of Theorem 1.1}

Let $v_{1}, \ldots, v_{2 \sigma}$ be the basis of $l\left(N_{p, \sigma}\right) \otimes k$ defined as in Section 2 associated to the strictly characteristic space $\mathcal{K}(X)$. The intersection matrix of $l\left(N_{p, \sigma} \otimes k\right)$ is $\left(\begin{array}{cc}0 & A \\ A^{t} & 0\end{array}\right)$, where $A$ is the matrix (3.1). For all $\alpha \in \operatorname{Aut}(X)$, each $v_{i}$ is an eigenvector of $\nu_{X}(\alpha)$. Assume $n$ is the non-symplectic index of $X$. Suppose $\nu_{X}(\alpha)\left(v_{1}\right)=\xi v_{1}$ and the order of $\xi$ in $k^{*}$ is $n$. Then $\nu_{X}(\alpha)\left(v_{i}\right)=F_{k}^{i-1}(\xi) v_{i}$ and $\xi$ determines $\nu_{X}(\alpha)$. Let $m$ be the smallest non-negative integer such that $F_{k}^{m}(\xi)=\xi^{-1}$. Because $F_{k}^{\sigma}(\xi)=\xi^{-1}$ (see Theorem 2.4), we know $m \leq \sigma$. If $m=0$, then $\xi=-1$ and $n=2$. If $m>0$, the order of $p$ in $(\mathbb{Z} / n)^{*}$ is $2 m$ and

$$
p^{m} \equiv p^{\sigma} \equiv-1 \quad(\bmod n)
$$

Therefore $m$ is a divisor of $\sigma$ and $\sigma / m$ is an odd integer. The order of $\nu_{X}(\alpha), n$ is a divisor of $p^{m}+1$. Because $\nu_{X}(\alpha) \in O\left(l\left(N_{p, \sigma}\right) \otimes k\right)$,

$$
a_{i}=v_{1} \cdot v_{\sigma+i+1}=\nu_{X}\left(v_{1}\right) \cdot \nu_{X}\left(v_{\sigma+i+1}\right)=\xi^{1-p^{i}} a_{i} .
$$

Hence $a_{i}=0$ unless $i$ is a multiple of $n=2 m$.

Conversely, assume $m$ is a divisor of $\sigma$ such that $\sigma / m$ is an odd integer. Assume $n=p^{m}+1$ and $\xi \in k^{*}$ is a primitive $n$-th root of unity. Suppose $a_{i}=0$ unless $i$ is a multiple of $2 m$. Let $g$ be a linear operator of $l\left(N_{p, \sigma}\right) \otimes k$ which sends $v_{i}$ to $F_{k}^{i-1}(\xi) v_{i}$ $(i=1, \ldots, 2 \sigma)$. It is clear that $g$ preserves the subspace $\mathcal{K}(X)$. Because $a_{i}=0$ for $2 m \nmid i$, we have $g\left(v_{i}\right) \cdot g\left(v_{j}\right)=v_{i} \cdot v_{j}$ for any $i$ and $j$, so $g \in O\left(l\left(N_{p, \sigma}\right) \otimes k\right)$. We claim that $g \in O\left(l\left(N_{p, \sigma}\right)\right)$, in other words, $g$ is rational over $\mathbb{F}_{p}$. Note that $g$ is rational over $\mathbb{F}_{p}$ if and only if $f(g(w))=g(f(w))$ for all $w \in l\left(N_{p, \sigma}\right) \otimes k$. For that, it is enough to check that $f\left(g\left(v_{i}\right)\right)=g\left(f\left(v_{i}\right)\right)$ for $i=1, \ldots, 2 \sigma$. Because $f\left(v_{i}\right)=v_{i+1}(i=1, \ldots, 2 \sigma-1)$,

$$
f\left(g\left(v_{i}\right)\right)=f\left(\xi^{p^{i-1}} v_{i}\right)=\xi^{p^{i}} v_{i+1}=g\left(v_{i+1}\right)=g\left(f\left(v_{i}\right)\right)
$$

for $i=1, \ldots, 2 \sigma-1$. We let $v^{\prime}=f\left(v_{2 \sigma}\right)$ and express $v^{\prime}=b_{1} v_{1}+b_{2} v_{2}+\cdots+b_{2 \sigma} v_{2 \sigma}$ $\left(b_{i} \in k\right)$. By the assumption, $v_{2 \sigma} \cdot v_{i}=0$ unless $i=\sigma-2 m e$ for a non-negative integer $e$ and $v_{2 \sigma} \cdot v_{\sigma}=1$. Since the pairing of $l\left(N_{p, \sigma}\right) \otimes k$ is defined over $\mathbb{F}_{p}$, for any $u, w \in l\left(N_{p, \sigma}\right) \otimes k$, we have $F_{k}(u \cdot w)=f(u) \cdot f(w)$. Hence $v^{\prime} \cdot v_{i}=0$ for $i \neq \sigma+1-2 m e(i \geq 2)$ and $v^{\prime} \cdot v^{\prime}=0$. It is straightforward to see that $b_{i}=0$ unless $i=1+2 m e$ and $g\left(v^{\prime}\right)=\xi v^{\prime}$, so

$$
f\left(g\left(v_{2 \sigma}\right)\right)=f\left(\xi^{p^{2 \sigma-1}} v_{2 \sigma}\right)=\xi^{p^{2 \sigma}} v^{\prime}=\xi v^{\prime}=g\left(f\left(v_{2 \sigma}\right)\right) .
$$

Therefore $g \in O\left(l\left(N_{p, \sigma}\right)\right)$ and, by Lemma 3.5, $g \in \operatorname{Im} \nu_{X}$. The non-symplectic index of $X$ is divisible by $p^{m}+1$.

Now we can see the non-symplectic index of $X$ is $p^{m}+1$ for $m=0$ or for a positive integer $m$ such that $\sigma / m$ is an odd integer. The non-symplectic index $N_{X}$ is exactly $p^{m}+1$ 
for a positive integer $m$ if and only if $a_{i}=0$ unless $2 m \nmid i$ and $a_{2 m j} \neq 0$ for some positive integer $j$. The number of possibly non-zero variables $a_{2 m e}$ is $(\sigma-m) /(2 m)$, so the set of all supersingular K3 surfaces of Artin invariant $\sigma$ and non-symplectic index $p^{m}+1$ form a $(\sigma-m) /(2 m)$-dimensional family. In particular, the non-symplectic index of $X$ is $p^{\sigma}+1$ if and only if $a_{i}=0$ for all $i$. Hence a supersingular K3 surface of Artin invariant $\sigma$ and of non-symplectic index $p^{\sigma}+1$ is unique up to isomorphism. This completes the proof of Theorem 1.1 .

Let us say the unique supersingular K3 surface of Artin invariant $\sigma$ with non-symplectic index is $p^{\sigma}+1$ is the special supersingular K3 surface of Artin invariant $\sigma$. Because a special supersingular K3 surface is defined over an algebraic closure of a finite field and it is unique, every special supersingular K3 surface has a model over a finite field. We will see some examples of special supersingular K3 surfaces below.

Corollary 4.1. The maximal value of the non-symplectic index of a K3 surface defined over $k$ is $p^{10}+1$. The only K3 surface with the maximal non-symplectic index is the special supersingular K3 surface of Artin invariant 10.

\section{Example}

Let $X$ be a complex algebraic K3 surface and $N_{X}$ be the non-symplectic index of $X$. The rank of $T(X)$ is divisible by $\phi\left(N_{X}\right)$. If the rank of $T(X)$ is equal to $\phi\left(N_{X}\right)$, then $X$ is a CM K3 surface and $X$ has a model over a number field (see [17, Corollary 3.9.4]). Many examples of K3 surfaces satisfying this condition are known (see [2,11,12]). Assume the rank of $T(X)$ is equal to $\phi(N)$ and $X$ is defined over a number field $F$. We choose $g \in \operatorname{Aut}(X)$ such that the order of $\rho_{X}(g)$ is $N_{X}$. For almost all finite places $v$ of $F, X$ has good reduction $X_{v}$ over $v$ and $g$ can be extended to $X_{v}$. For such a $v$, the height (and the Artin invariant) of $X_{v}$ is determined by the congruence class of the residue characteristic $p_{v}$ of $v$ modulo $N_{X}$. In particular, if $m$ is the smallest positive integer such that $p_{v}^{m} \equiv-1$ modulo $N_{X}$, then $X_{v}$ is a supersingular of Artin invariant $m$ (see [9, Theorem 4.7], 8, Theorem 2.3]). Moreover, in this case, $\nu_{X_{v}}(g) \in O\left(l\left(N_{p, m}\right)\right)$ has $2 m$ distinct eigenvalues, so $a_{i}=0$ for all $i$ in (3.1) and $X_{v}$ is a special supersingular K3 surface of Artin invariant $m$.

For example, a sextic double cover K3 surface

$$
X_{50}: w^{2}=x^{6}+x y^{5}+y z^{5}
$$

has a purely non-symplectic automorphism of order 50 ,

$$
\alpha:(x, y, z, w) \mapsto\left(x, \xi_{50}^{40} y, \xi_{50}^{2} z,-w\right) .
$$


Here $\xi_{50}$ is a primitive 50-th root of unity. If a rational prime $p$ does not divide 50, $X_{50}$ has good reduction over $\overline{\mathbb{Z} / p}$ and $\alpha$ is extended to the reduction. Let $\left(X_{50, p}, \alpha_{p}\right)$ be the reduction of $(X, \alpha)$ over $\overline{\mathbb{Z} / p}$. In the following cases, the reduction $X_{p}$ is a special supersingular K3 surface.

\begin{tabular}{|c|c|}
\hline$p(\bmod 50)$ & Artin invariant \\
\hline 49 & 1 \\
\hline 7,43 & 2 \\
\hline $9,19,29,39$ & 5 \\
\hline $3,13,17,23,27,33,37,47$ & 10 \\
\hline
\end{tabular}

Table 5.1

Because many complex K3 surfaces, for which the rank of transcendental lattice is equal to the $\phi$ value of the non-symplectic index, are defined over $\mathbb{Q}$, many special supersingular K3 surfaces have models over a prime field $\mathbb{F}_{p}$. We have the following question naturally.

Question 5.1. Does every special supersingular K3 surface have a model over a prime field?

\section{Acknowledgments}

The author is grateful to the anonymous referee, of whom suggestions was very helpful to improve the exposition. This research was supported by Basic Science Research Program through the National Research Foundation of Korea (NRF) funded by the Ministry of Education, Science and Technology [2018R1D1A1B07044995].

\section{References}

[1] D. Bragg and M. Lieblich, Twistor spaces for supersingular K3 surfaces, arXiv:1804.07282v5.

[2] S. Brandhorst, How to determine a K3 surface from a finite automorphism, arXiv:1604.08875.

[3] _ Automorphisms of Salem degree 22 on supersingular K3 surfaces of higher Artin invariant - a short note, arXiv:1609.02348.

[4] H. Esnault and K. Oguiso, Non-liftability of automorphism groups of a K3 surface in positive characteristic, Math. Ann. 363 (2015), no. 3-4, 1187-1206. 
[5] H. Esnault, K. Oguiso and X. Yu, Automorphisms of elliptic K3 surfaces and Salem numbers of maximal degree, Algebr. Geom. 3 (2016), no. 4, 496-507.

[6] L. Illusie, Complexe de de Rham-Witt et cohomologie cristalline, Ann. Sci. École Norm. Sup. (4) 12 (1979), no. 4, 501-661.

[7] J. Jang, Representations of the automorphism group of a supersingular $k 3$ surface of Artin-invariant 1 over odd characteristic, J. Chungcheong Math. Soc. 27 (2014), no. 2, 287-295.

[8] _ Some remarks on non-symplectic automorphisms of K3 surfaces over a field of odd characteristic, East Asian Math. J. 30 (2014), no. 3, 321-326.

[9] - The representations of the automorphism groups and the Frobenius invariants of K3 surfaces, Michigan Math. J. 65 (2016), no. 1, 147-163.

[10] A lifting of an automorphism of a K3 surface over odd characteristic, Int. Math. Res. Not. IMRN 2017 (2017), no. 6, 1787-1804.

[11] J. Keum, Orders of automorphisms of K3 surfaces, Adv. Math. 303 (2016), 39-87.

[12] S. Kondō, Automorphisms of algebraic K3 surfaces which act trivially on Picard groups, J. Math. Soc. Japan 44 (1992), no. 1, 75-98.

[13] V. V. Nikulin, Integral symmetric bilinear forms and some of their applications, Izv. Akad. Nauk SSSR Ser. Mat. 43 (1979), no. 1, 111-177.

[14] N. O. Nygaard, Higher de Rham-Witt complexes of supersingular K3 surfaces, Compositio Math. 42 (1980), no. 2, 245-271.

[15] A. Ogus, Supersingular K3 crystals, in: Journées de Géométrie Algébrique de Rennes (Rennes, 1978), Vol. II, 3-86, Astérisque 64, Soc. Math. France, Paris, 1979.

[16] _ A crystalline Torelli theorem for supersingular K3 surfaces, in: Arithmetic and Geometry, Vol. II, 361-394, Progr. Math. 36, Birkhäuser Boston, Boston, MA, 1983.

[17] J. Rizov, Kuga-Satake abelian varieties of K3 surfaces in mixed characteristic, J. Reine Angew. Math. 648 (2010), 13-67.

[18] M. Schütt, Dynamics on supersingular K3 surfaces, Comment. Math. Helv. 91 (2016), no. $4,705-719$.

[19] I. Shimada, Automorphisms of supersingular K3 surfaces and Salem polynomials, Exp. Math. 25 (2016), no. 4, 389-398. 
[20] X. Yu, Elliptic fibrations on K3 surfaces and Salem numbers of maximal degree, J. Math. Soc. Japan 70 (2018), no. 3, 1151-1163.

Junmyeong Jang

Department of Mathematics, University of Ulsan, Daehakro 93, Namgu Ulsan 44610, Korea

E-mail address: jmjang@ulsan.ac.kr 